\title{
Desidratação, acidente vascular cerebral e disfagia: revisão sistemática da literatura
}

\section{Dehydration, stroke and dysphagia: systematic review}

\author{
Marina Santos Tupi Barreira Schettino ${ }^{1}$ (D), Déborah Carollina Costa Silva ${ }^{2}$ (1), \\ Nayara Aparecida Vasconcelos Pereira-Carvalho' (1), Laelia Cristina Caseiro Vicente ${ }^{3}$ (1), \\ Amélia Augusta de Lima Friche ${ }^{3}$ (1)
}

\section{RESUMO}

Objetivos: trata-se de revisão sistemática da literatura científica sobre a associação entre o acidente vascular cerebral, desidratação e disfagia orofaríngea. Estratégia de pesquisa: o levantamento bibliográfico foi realizado nas bases de dados científicos: MEDLINE, LILACS, SciELO, Web of Science e Cochrane. Critérios de seleção: foram incluídos os estudos que preencheram os seguintes critérios de inclusão: ser artigo original, com resumo disponível; ter sido publicado entre os anos de 2001 e 2018 e nos idiomas português, inglês ou espanhol; abordar o tema desidratação em indivíduos após acidente vascular cerebral. A triagem e análise dos estudos foram realizadas por dois avaliadores independentes. Resultados: dentre os 484 artigos localizados para a triagem, 43 foram selecionados para leitura completa e 18 foram incluídos na análise final. Foram descritos diferentes métodos de avaliação do estado de hidratação nos indivíduos após acidente vascular cerebral, tais como: análise da relação BUN /creatinina, osmolaridade plasmática, relação ureia/creatinina, gravidade específica da urina, coloração da urina, ingestão hídrica, balanço hídrico, bioimpedância elétrica, avaliação clínica, análise de eletrólitos isolados. A prevalência de desidratação em pacientes após acidente vascular cerebral, durante a internação, variou de $11 \%$ a $66 \%$ e está associada à gravidade e piora na evolução clínica. Conclusão: foi possível compreender a complexidade do processo de mensuração do estado de hidratação em indivíduos após acidente vascular cerebral e sua associação com a disfagia. Estudos enfocando essa temática são de extrema relevância, visto a sua influência sobre a taxa de mortalidade e morbidade nesta população.

Palavras-chave: Desidratação; Hidratação; Acidente vascular cerebral; Transtornos de deglutição; Água corporal

\begin{abstract}
Purpose: this is a systematic review of scientific literature associated with stroke, dehydration and oropharyngeal dysphagia (OD). Research strategy: bibliographic survey was conducted in scientific databases: MEDLINE, LILACS, SciELO, Web of Science and Cochrane. Selection criteria: studies that met the following inclusion criteria were included: being original article with summary available; have been published in the last seventeen years (2001-2018) and in Portuguese, English or Spanish languages; and addressing dehydration in individuals after stroke Screening and analysis of the studies were performed by two independent evaluators. Results: among the 484 articles found for screening, 43 were selected for full reading and 18 articles were included in the final analysis. Different methods of assessing hydration status have been described in individuals post-stroke, such as ratio analysis blood urea nitrogen $(\mathrm{BUN}) /$ creatinine, plasma osmolality, urea / creatinine, urine specific gravity, urine color, water intake, water balance, bioelectrical impedance analysis (BIA), clinical evaluation and analysis of electrolytes. The prevalence of dehydration in post-stroke during hospitalization varied from $11 \%$ to $66 \%$ and is associated with severity and deterioration in the clinical evolution. Conclusion: It was possible to understand the complexity of the measurement of hydration status in individuals after stroke and its association with dysphagia. Studies focusing on the association between dehydration and stroke are very important, due to its influence on mortality and morbidity in this population.
\end{abstract}

Keywords: Dehydration; Hydration; Stroke; Swallowing disorder; Body water

\footnotetext{
Trabalho realizado no Programa de Pós-graduação em Ciências Fonoaudiológicas, Universidade Federal de Minas Gerais - UFMG - Belo Horizonte (MG), Brasil. ${ }^{1}$ Mestrado em Ciências Fonoaudiológicas, Faculdade de Medicina, Universidade Federal de Minas Gerais - UFMG - Belo Horizonte (MG), Brasil.

${ }^{2}$ Curso de Fonoaudiologia, Faculdade de Medicina da Universidade Federal de Minas Gerais - UFMG - Belo Horizonte (MG), Brasil. ${ }^{3}$ Departamento de Fonoaudiologia, Faculdade de Medicina, Universidade Federal de Minas Gerais - UFMG - Belo Horizonte (MG), Brasil.

Conflitos de interesses: Não.

Contribuição dos autores: MSTBS foi responsável pela coleta e tabulação dos dados, análise dos resumos e artigos e elaboração do manuscrito; DCCS foi responsável pela coleta e tabulação dos dados, análise dos resumos e artigos e elaboração do manuscrito; NAVPC foi responsável pela coleta dos dados, análise dos resumos e elaboração do manuscrito; LCCV foi responsável pela orientação do projeto e delineamento do estudo e correção do manuscrito; AALF foi responsável pela orientação do projeto, concepção e delineamento do estudo e correção do manuscrito.

Financiamento: Nada a declarar.
}

Autor correspondente: Marina Santos Tupi Barreira Schettino. E-mail: marinabarreirafono@yahoo.com.br

Recebido: Agosto 15, 2019; Aceito: Outubro 21, 2019 


\section{INTRODUÇÃO}

A água é o principal constituinte das células, tecidos e órgãos dos seres humanos, sendo que o estado de hidratação adequado é essencial para manter a homeostase celular e o funcionamento dos órgãos e sistemas ${ }^{(1)}$.

Em indivíduos saudáveis, mesmo as alterações leves no estado de hidratação são capazes de comprometer as funções cognitivas, o estado de alerta e a capacidade de realizar atividades físicas ${ }^{(1-4)}$.

Dentre alguns dos sinais e sintomas relacionados à desidratação, estão confusão mental, alterações cognitivas, ressecamento de mucosas, hipotensão ortostática e taquicardia ${ }^{(1,2,5)}$. Limitações nas funções motoras e no padrão de deglutição, comorbidades frequentemente encontradas em indivíduos após acidente vascular cerebral (AVC), também contribuem para maior vulnerabilidade para a desidratação ${ }^{(6,7)}$.

A disfagia orofaríngea é caracterizada como um distúrbio na dinâmica da deglutição, com prevalência em indivíduos após AVC, acima de $37 \% \%^{(8,9)}$. É comum que indivíduos com disfagia orofaríngea sejam expostos a restrições alimentares e na ingestão de líquidos, modificações na consistência e/ou ao uso de via alternativa de alimentação, até que estejam aptos a receber a dieta por via oral ${ }^{(10)}$.

A inabilidade de ingestão segura e eficaz de líquidos e alimentos, o comprometimento do estado de alerta e da capacidade de acessar os líquidos podem ser consideradas condições de risco para o estado de hidratação do indivíduo após $\mathrm{AVC}^{(10)}$, porém, a relação potencial entre desidratação, AVC e disfagia ainda é pouco discutida na literatura ${ }^{(8)}$.

Diretrizes clínicas enfatizam a importância da adequada hidratação após o AVC, uma vez que a desidratação pode aumentar a viscosidade sanguínea e ocasionar a diminuição do fluxo intravascular ${ }^{(11)}$. Além disso, a desidratação está associada ao aumento do risco de tromboembolismo venoso e com a piora na evolução clínica durante a internação hospitalar ${ }^{(12)}$.

Algumas técnicas são descritas como utilizadas para a avaliação do estado de hidratação de indivíduos após AVC, tais como técnicas não invasivas (mensuração da ingestão hídrica e análise da perda de peso corporal, dos sinais vitais e do estado da pele e mucosas); exames laboratoriais (osmolaridade plasmática, osmolaridade urinária, relação BUN(blood urea nitrogen)/creatinina e ureia/creatinina) e análise da impedância bioelétrica ${ }^{(12)}$ Outras técnicas e biomarcadores também são utilizados, porém não há consenso na literatura quanto ao padrão ouro ${ }^{(6-8,13,14)}$.

\section{OBJETIVO}

O objetivo deste estudo foi sistematizar as principais pesquisas que avaliam o estado de desidratação em pacientes pós-AVC e apresentar os principais resultados obtidos a partir destes trabalhos, contribuindo para a melhor compreensão da relação entre AVC e desidratação e possíveis associações com a disfagia orofaríngea.

\section{ESTRATÉGIA DE PESQUISA}

Trata-se de revisão sistemática da literatura científica sobre desidratação e disfagia em pacientes pós-AVC, em que a metodologia baseou-se em estudos anteriores e recomendações sobre o tema ${ }^{(15,16)}$.

A primeira etapa de levantamento bibliográfico foi realizada nos portais: PubMed, para acesso aos periódicos indexados no MEDLINE; Biblioteca Regional de Medicina (BIREME), para acesso às bases de dados científicos Literatura Latino/americana e do Caribe em Ciências da Saúde (LILACS) e Scientific Electronic Library On Line (SciELO); Web of Knowledge, para acesso à base de dados científicos Web of Science; Cochrane. Utilizaram-se os seguintes termos relacionados à hidratação corporal (no PubMed: dehydration [Title/Abstract] OR desidratação [Title/Abstract] OR desidratación [Title/Abstract] OR hydration [Title/Abstract] OR hidratação [Title/Abstract] OR hidratación [Title/Abstract]); à alteração neurológica (no PubMed: stroke [Title/Abstract] OR acidente vascular cerebral [Title/Abstract] OR accidente cerebrovascular [Title/Abstract]) e à alteração no padrão de deglutição (no PubMed: deglutition disorders [Title/Abstract] OR transtornos de deglutição [Title/Abstract]) OR transtornos de deglutición [Title/Abstract]). As estratégias foram adaptadas para cada base de dados, devido às diferenças nos mecanismos de busca e nos termos presentes em cada base. Para análise dos artigos do MEDLINE, optou-se por realizar duas pesquisas: uma em que se incluíram termos relacionados à hidratação corporal e alteração neurológica e outra, incluindo os termos relacionados à alteração no padrão de deglutição.

\section{CRITÉRIOS DE SELEÇÃO}

A segunda etapa compreendeu a triagem e análise dos estudos e foi realizada por dois avaliadores, de forma independente, com base no título e resumo dos artigos. Foram incluídos os estudos que preencheram os seguintes critérios de inclusão: ser artigo original, com resumo disponível; ter sido publicado entre os anos de 2001 e 2018 e nos idiomas português, inglês ou espanhol e abordar o tema desidratação em indivíduos após acidente vascular cerebral.

Os resultados dos avaliadores foram comparados e os artigos que obtiveram respostas "sim" e/ou "talvez", por parte dos dois avaliadores, foram incluídos na análise; a existência de dois "não" excluiu o artigo da análise. Em caso de discordância, realizou-se reunião de consenso e, quando necessário, solicitou-se a participação de um terceiro avaliador.

\section{ANÁLISE DOS DADOS}

Para os estudos selecionados na triagem, realizou-se leitura dos artigos na íntegra e inclusão de questões, como amostra composta por adultos com idade superior a 18 anos e apresentar, na metodologia, critérios definidos sobre o processo de avaliação do estado de hidratação em pacientes após AVC. O fluxograma de seleção/inclusão dos artigos encontrados, selecionados para triagem, leitura na íntegra e incluídos para análise, por fonte de dados, está descrito na Figura 1. 


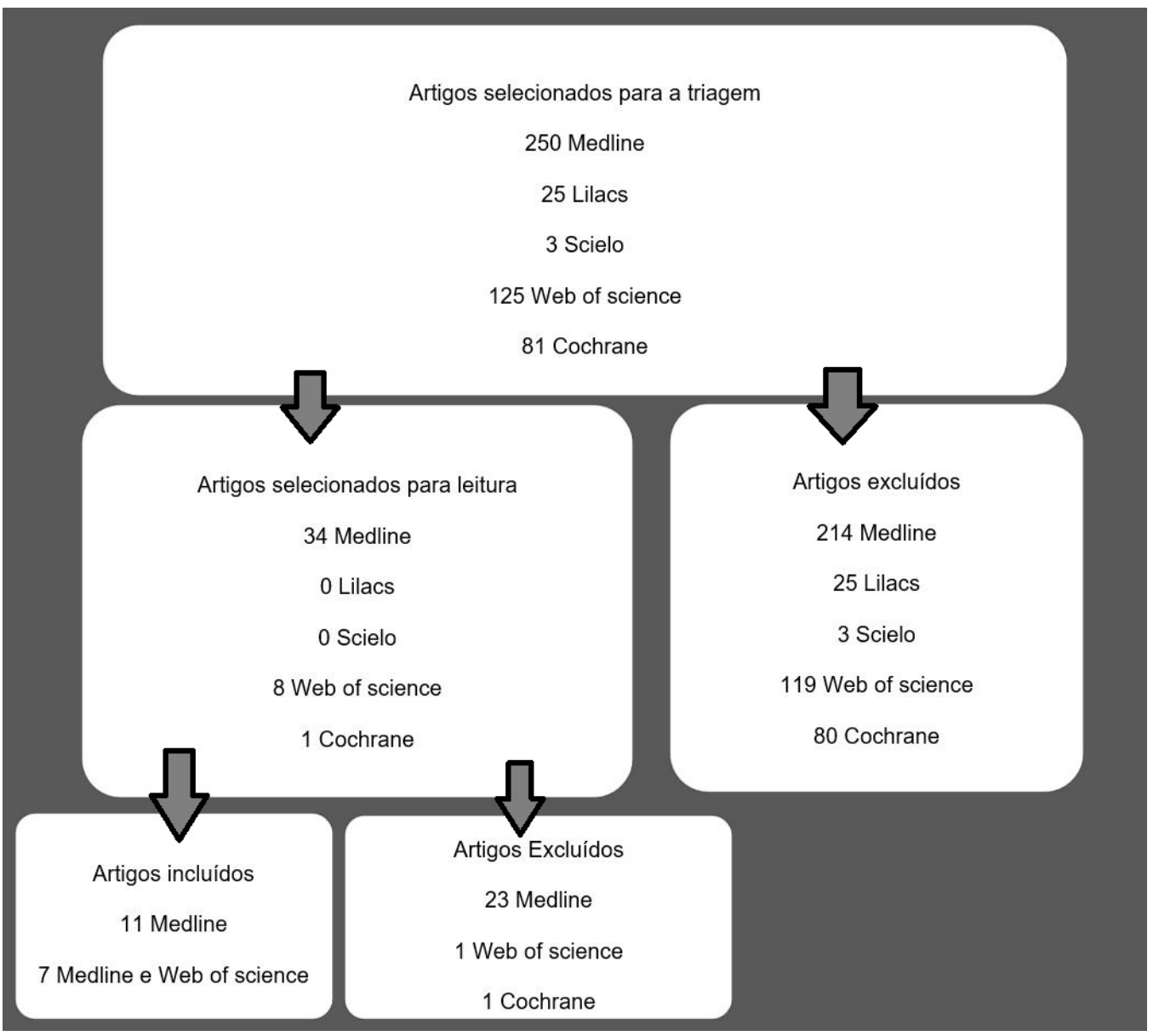

Figura 1. Fluxograma de seleção dos artigos incluídos na revisão sistemática da literatura

Após a seleção dos artigos, realizou-se coleta dos seguintes dados relevantes para a análise: base de dados pesquisada; nome do artigo; primeiro autor; ano de publicação; desenho do estudo; objetivo do estudo; tamanho amostral; método de avaliação utilizado; perfil dos pacientes incluídos no estudo e resultados obtidos (Quadro 1).

Com o objetivo de avaliar a concordância dos avaliadores durante a seleção dos artigos, utilizou-se a análise de concordância por meio da estatística Kappa, resultando em valor 0,9, confiabilidade considerada excelente ${ }^{(30)}$.

\section{RESULTADOS}

Dentre os 484 artigos localizados para a triagem, 43 foram selecionados para leitura completa e 18 foram incluídos na análise final ${ }^{(6-8,12,14,17-29)}$. Os artigos excluídos não avaliavam o estado de hidratação ou de deglutição de pacientes internados após acidente vascular cerebral, ou a faixa etária dos sujeitos da pesquisa não se enquadrava aos critérios de inclusão estabelecidos. A amostra final incluiu 4 artigos ${ }^{(12,27-29)}$ publicados entre 2001 e 2004, $10 \operatorname{artigos}^{(6-8,14,21-26)}$ entre 2005 e $2013,2 \operatorname{artigos}^{(19,20)}$ de 2016 e $2 \operatorname{artigos}^{(17,18)}$ de 2018.

Todos os artigos incluídos na análise final foram pesquisados na base de dados MEDLINE, sendo que $41 \%$ deles também estavam indexados na base Web of Science. Das 18 publicações,
$100 \%$ estavam em inglês. Não foram encontradas publicações em espanhol ou em português compatíveis com os critérios de inclusão estabelecidos para o estudo. Nenhum estudo selecionado foi realizado no Brasil ou em países latino-americanos.

O tamanho da amostra variou entre 13 e 2591 indivíduos, sendo que, em 5 estudos ${ }^{(8,22,23,25,28)}$, a amostra foi composta apenas por indivíduos que apresentavam o primeiro diagnóstico de AVC. O histórico de AVC prévio não foi definido como critério de exclusão dos pacientes, em $72 \%$ dos estudos ${ }^{(6,7,12,14,17-21,24,26,27,29)}$. Em 9 estudos, a amostra foi composta apenas por AVC isquêmico ${ }^{(6-8,12,14,17,19,22,25)}$, em 3, por AVC isquêmico e hemorrágico ${ }^{(18,24,28)}$ e 6 estudos não especificaram o tipo de $\operatorname{AVC}^{(20,21,23,26,27,29)}$.

Em relação ao desenho dos estudos selecionados, observou-se que os 18 estudos eram observacionais, sendo que 12 eram prospectivos $^{(7,8,12,20-25,27-29)}$ e 6 , retrospectivos ${ }^{(6,14,17-19,26)}$.

Poucos estudos conduziram uma análise comparativa entre disfagia orofaríngea e desidratação. Em $33 \%$ deles $^{(6,7,12,14,25,26)}$, não foi realizada avaliação da deglutição e, em $17 \%^{(21,23,24)}$, os autores não especificaram o método de avaliação utilizado. Nos estudos em que a avaliação foi realizada $(\mathrm{n}=9)^{(8,17-20,22,27-29)}$, verificou-se o uso de avaliação clínica associada à videofluoroscopia $(n=4)^{(8,20,27,29)}$ ou apenas a realização da avaliação clínica à beira do leito $(\mathrm{n}=5)^{(17-19,22,28)}$.

Foram descritos diferentes métodos de avaliação do estado de hidratação nos indivíduos após AVC, tais como análise da relação BUN/creatinina $(\mathrm{n}=10)^{(6-8,17-20,25-27)}$, osmolaridade plasmática $(n=4)^{(12,14,21,26)}$, relação ureia/creatinina $(n=3)^{(12,23,24)}$, 


\begin{tabular}{|c|c|c|c|c|c|c|}
\hline 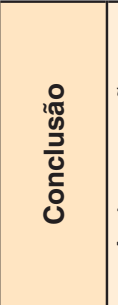 & 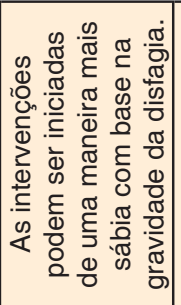 & 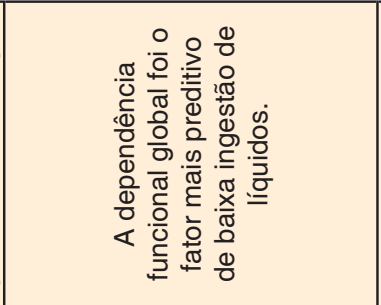 & 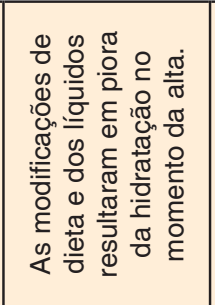 & 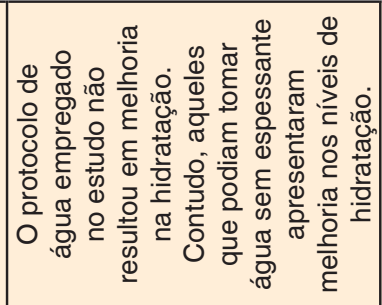 & 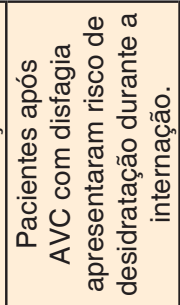 & 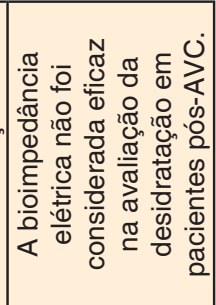 \\
\hline 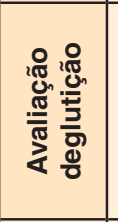 & 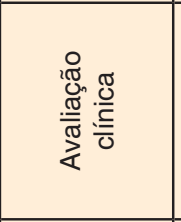 & 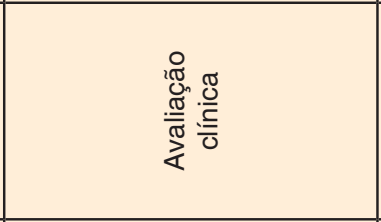 & 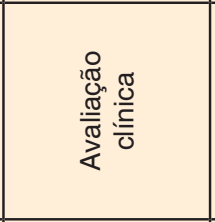 & 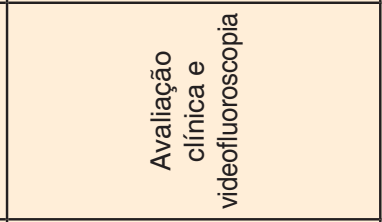 & 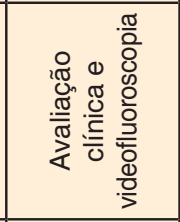 & 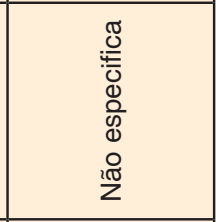 \\
\hline 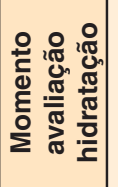 & 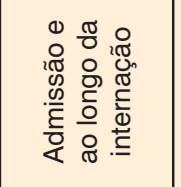 & 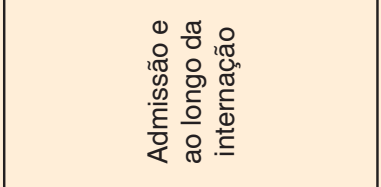 & 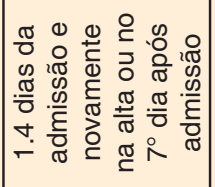 & 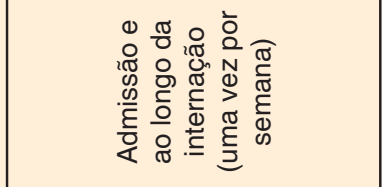 & 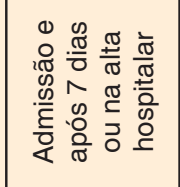 & 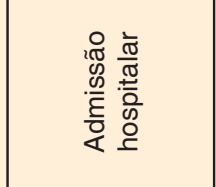 \\
\hline 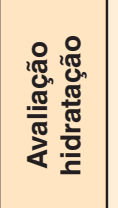 & 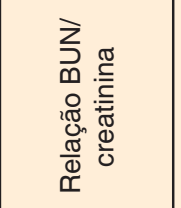 & 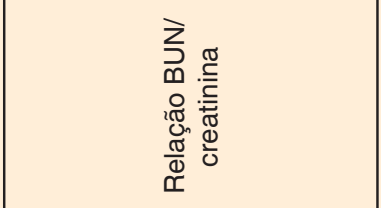 & 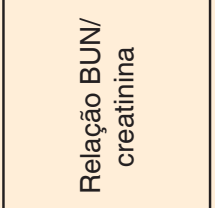 & 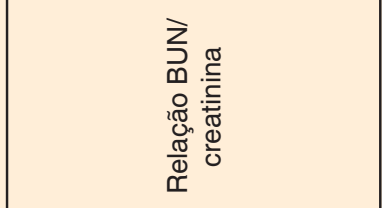 & 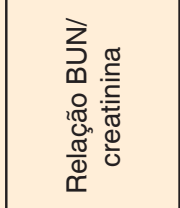 & 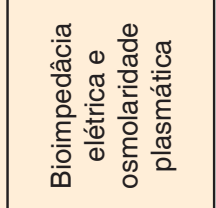 \\
\hline $\begin{array}{l}0 \\
\stackrel{2}{⿺} \\
\stackrel{0}{2}\end{array}$ & $\begin{array}{l}.8 \\
\stackrel{8}{E} \\
\stackrel{0}{0} \\
\overline{0} \\
\underline{0} \\
\underline{0}\end{array}$ & 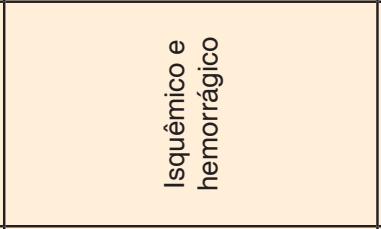 & 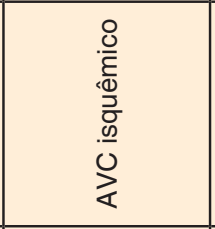 & 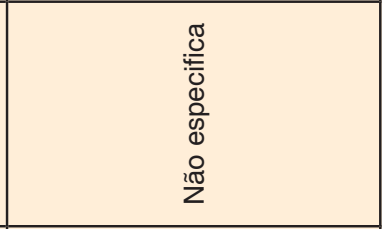 & 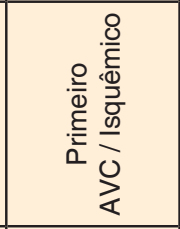 & 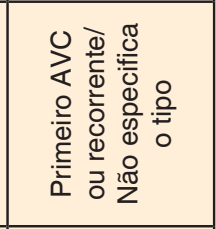 \\
\hline 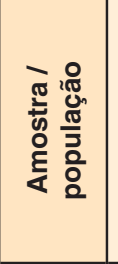 & $\underset{0}{\mathbb{N}}$ & 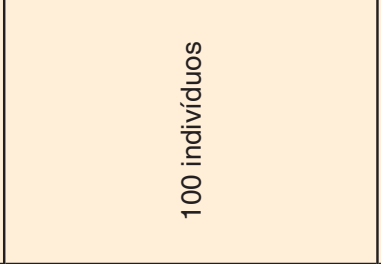 & 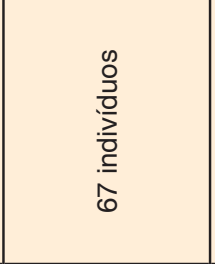 & 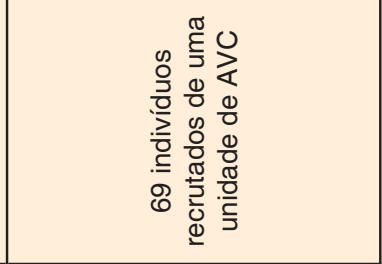 & 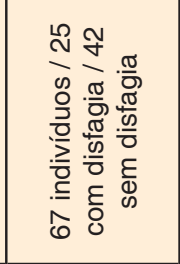 & 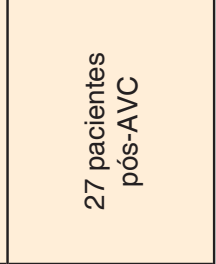 \\
\hline$\frac{\stackrel{0}{0}}{\frac{\grave{Z}}{0}}$ & 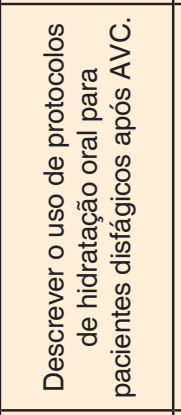 & 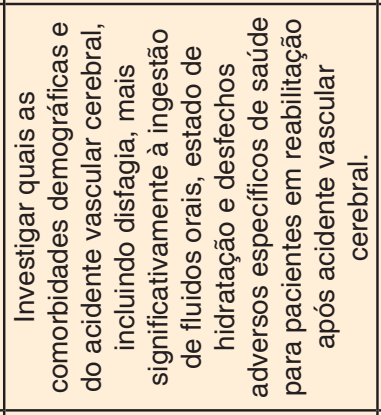 & 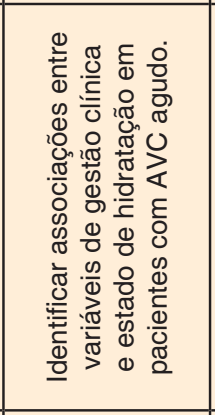 & 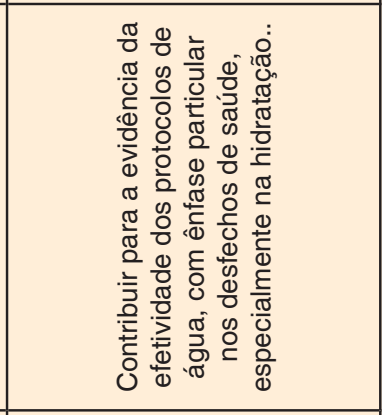 & 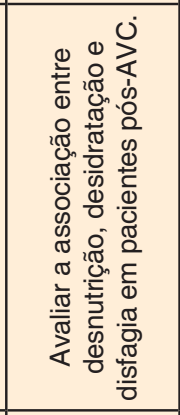 & 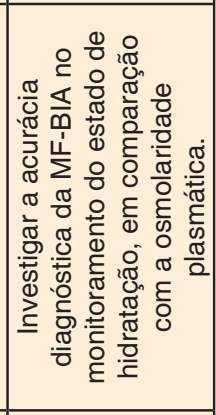 \\
\hline 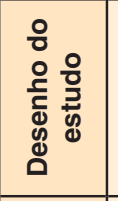 & 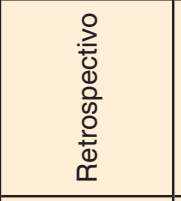 & 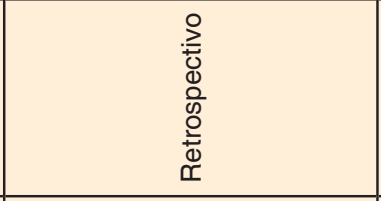 & 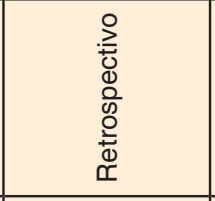 & 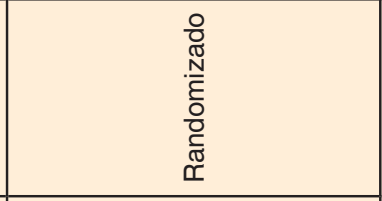 & 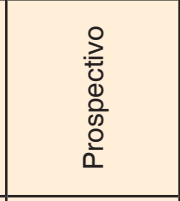 & 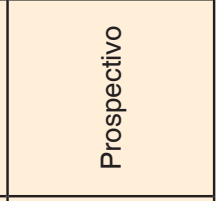 \\
\hline 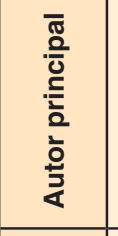 & 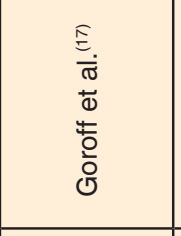 & 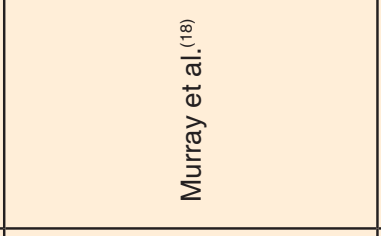 & 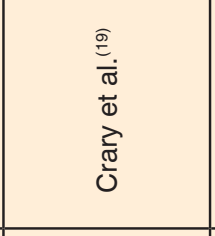 & 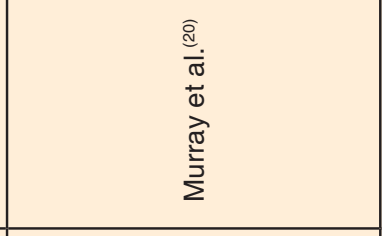 & 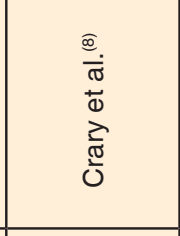 & 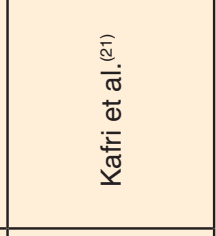 \\
\hline$\stackrel{\circ}{\frac{\varrho}{4}}$ & 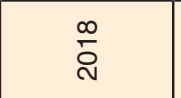 & $\stackrel{\infty}{\stackrel{N}{N}}$ & $\stackrel{0}{\stackrel{N}{N}}$ & $\stackrel{0}{\stackrel{N}{N}}$ & $\stackrel{m}{N}^{\infty}$ & $\stackrel{m}{i}$ \\
\hline
\end{tabular}




\begin{tabular}{|c|c|c|c|c|c|c|c|}
\hline 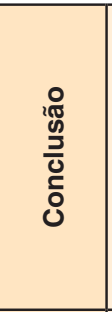 & 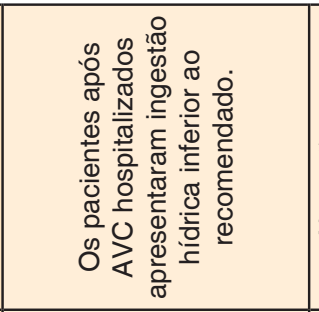 & 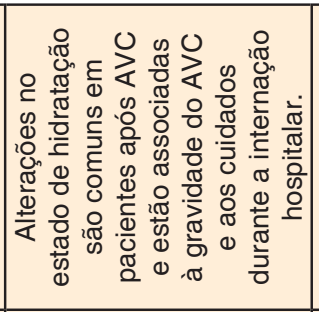 & 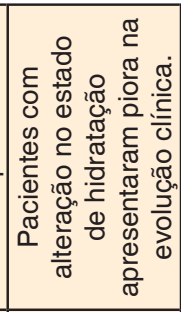 & 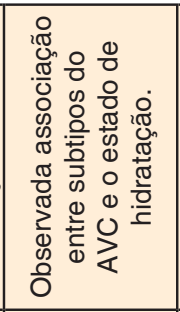 & 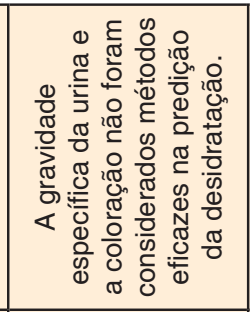 & 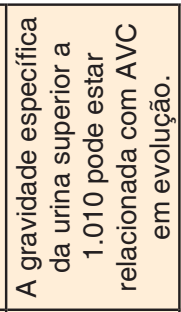 & 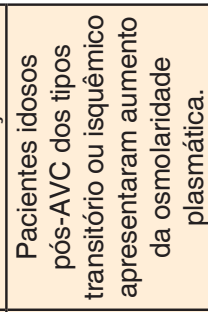 \\
\hline 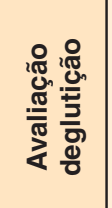 & 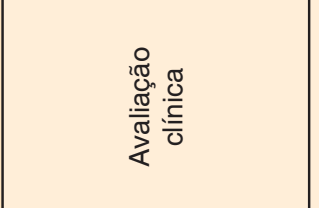 & 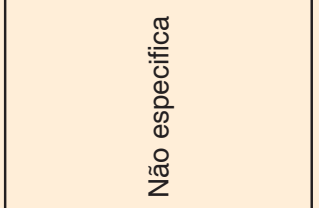 & 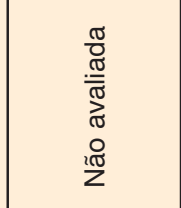 & $\begin{array}{l}\frac{\pi}{0} \\
\frac{.0}{\pi} \\
\frac{\pi}{\pi} \\
0 \\
\frac{\pi}{2} \\
\frac{\pi}{2}\end{array}$ & 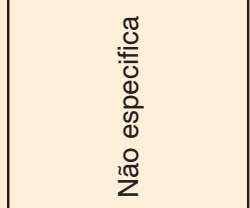 & $\begin{array}{l}\frac{\pi}{0} \\
\frac{\sigma}{\pi} \\
\frac{\pi}{\pi} \\
0 \\
\frac{0}{2} \\
\frac{\pi}{2}\end{array}$ & 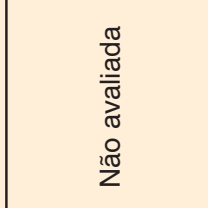 \\
\hline 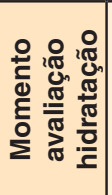 & 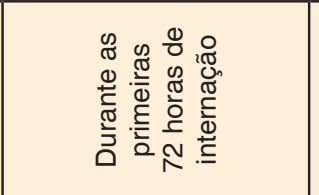 & 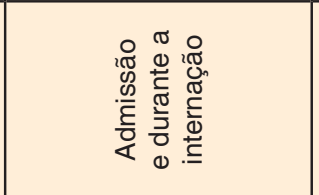 & 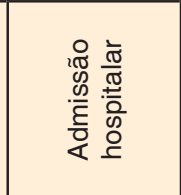 & 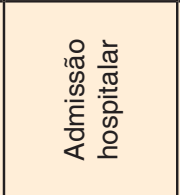 & 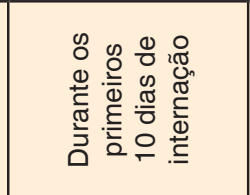 & 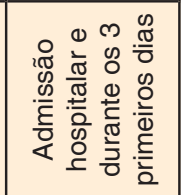 & 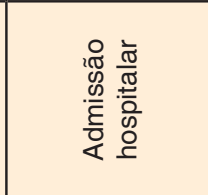 \\
\hline 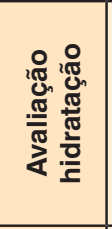 & 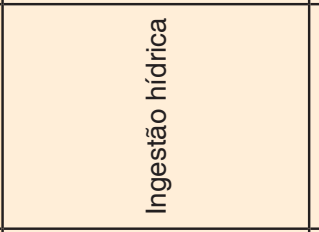 & 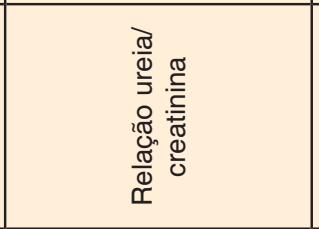 & 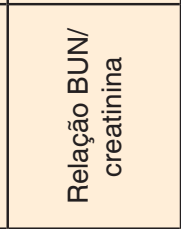 & 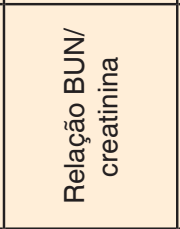 & 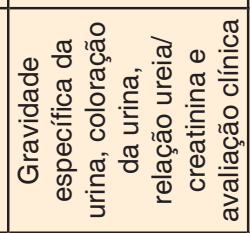 & 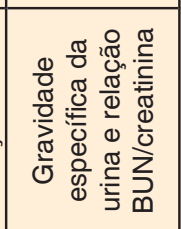 & 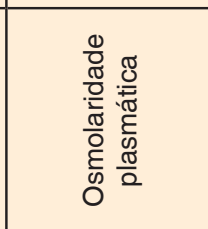 \\
\hline $\begin{array}{l}0 \\
\stackrel{0}{a} \\
\circ \\
\stackrel{0}{2}\end{array}$ & 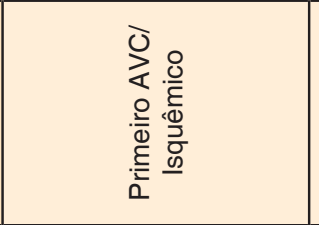 & 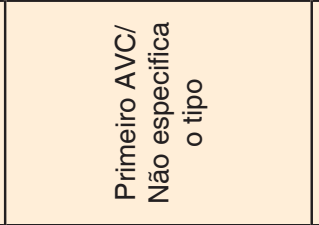 & 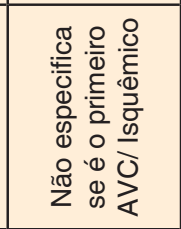 & 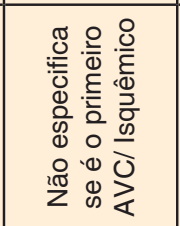 & 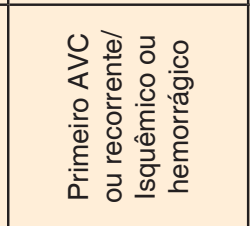 & 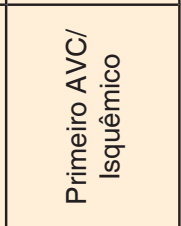 & 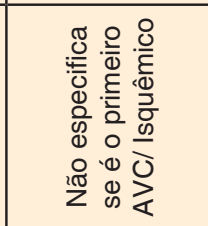 \\
\hline 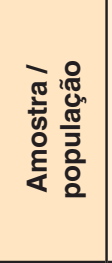 & 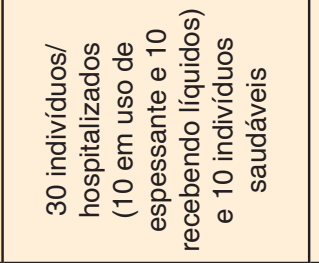 & 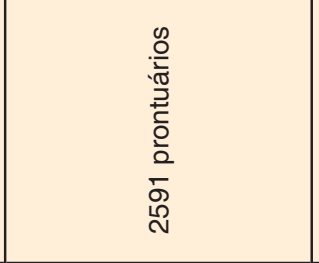 & 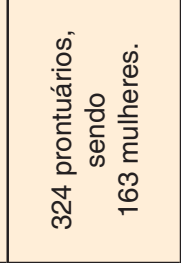 & 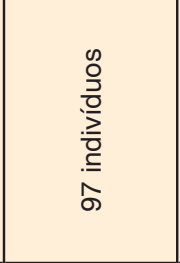 & 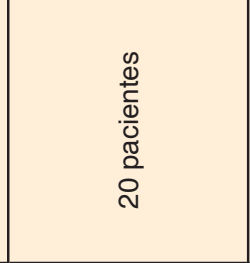 & 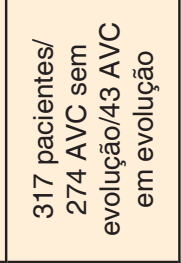 & 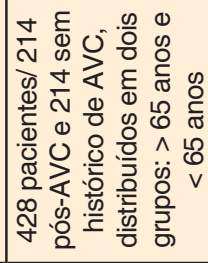 \\
\hline$\frac{\stackrel{0}{0}}{\stackrel{0}{\frac{1}{0}}}$ & 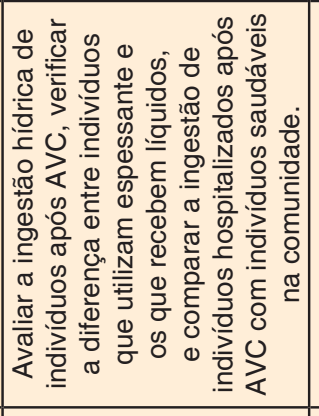 & 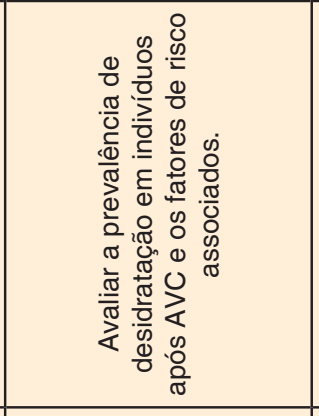 & 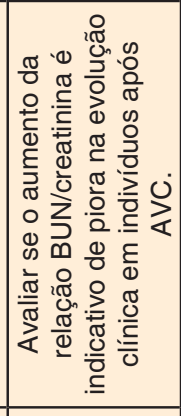 & 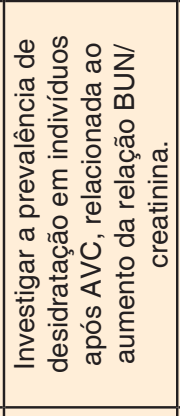 & 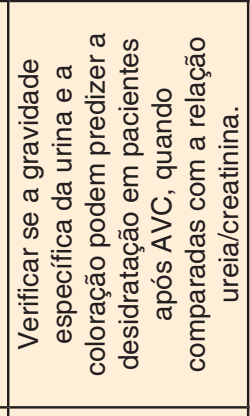 & 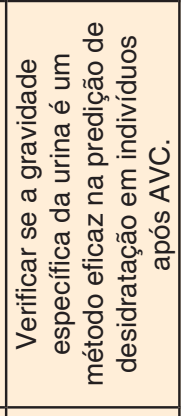 & 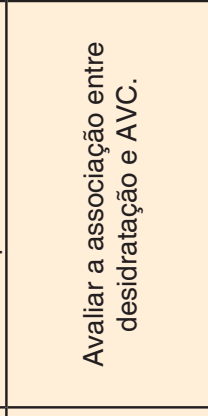 \\
\hline 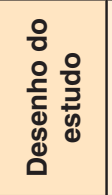 & 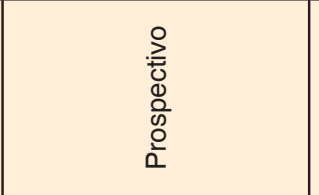 & 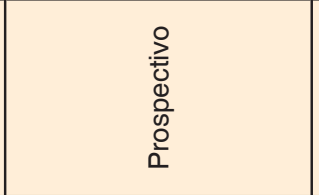 & 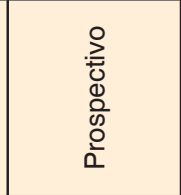 & 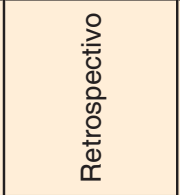 & 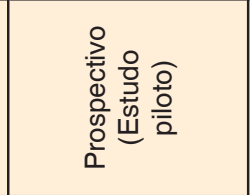 & 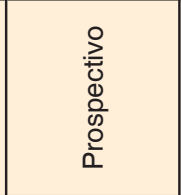 & 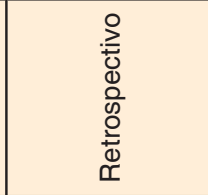 \\
\hline 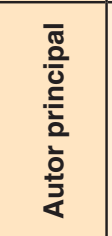 & 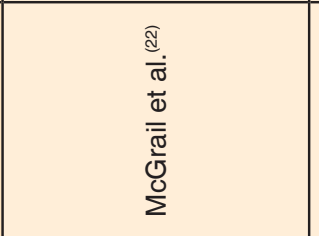 & 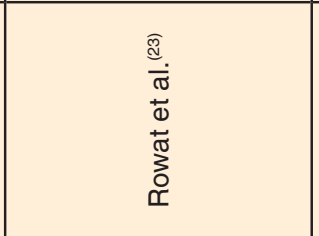 & 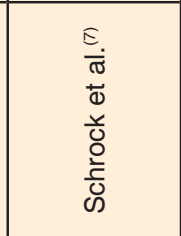 & 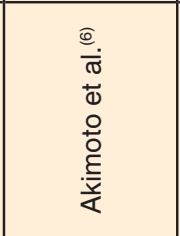 & 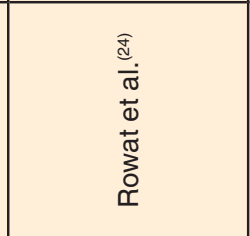 & 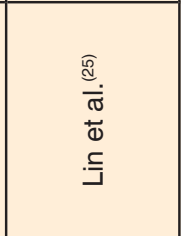 & 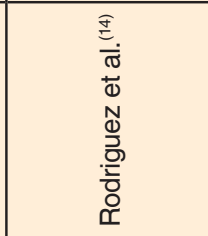 \\
\hline$\stackrel{\circ}{\frac{\varrho}{4}}$ & $\underset{\sim}{\sim}$ & $\stackrel{\sim}{\stackrel{N}{N}}$ & $\stackrel{N}{\sim}$ & $\bar{N}$ & $\bar{i}$ & $\bar{\sim}$ & ¿্̀ \\
\hline
\end{tabular}




\begin{tabular}{|c|c|c|c|c|c|}
\hline 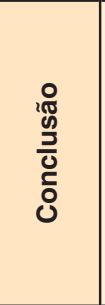 & 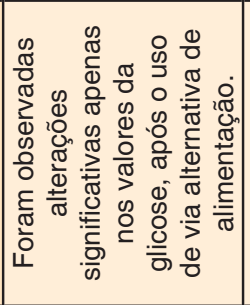 & 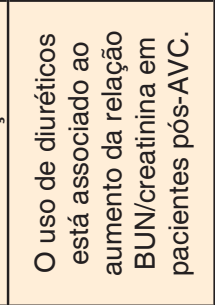 & 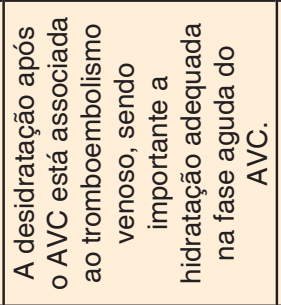 & 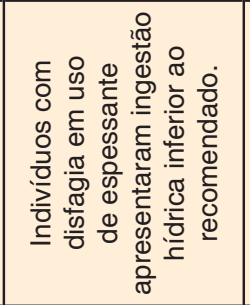 & 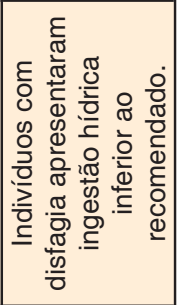 \\
\hline 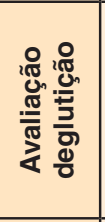 & $\begin{array}{l}\frac{\pi}{0} \\
\frac{0}{\pi} \\
\frac{0}{\pi} \\
\frac{\pi}{\pi} \\
2 \\
\frac{\pi}{2 \pi} \\
2\end{array}$ & 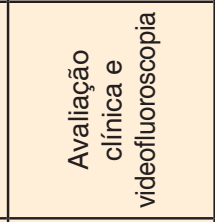 & $\begin{array}{l}\frac{\pi}{0} \\
\frac{\pi}{\pi} \\
\frac{\pi}{\pi} \\
0 \\
\frac{\pi}{2} \\
\frac{\pi}{2}\end{array}$ & 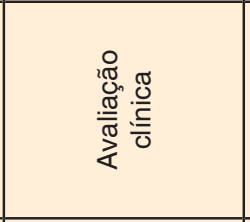 & 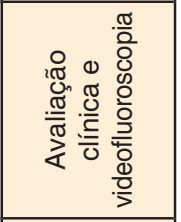 \\
\hline 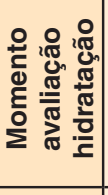 & 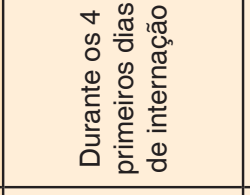 & 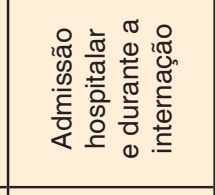 & 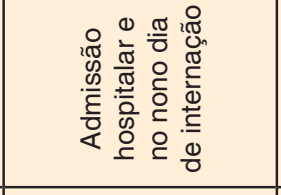 & 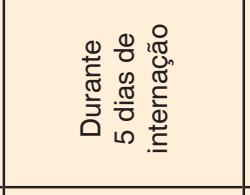 & 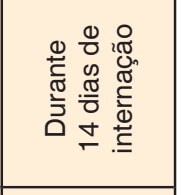 \\
\hline 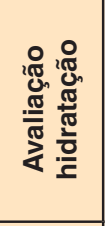 & 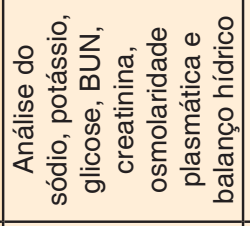 & 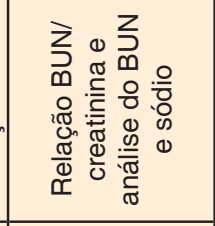 & 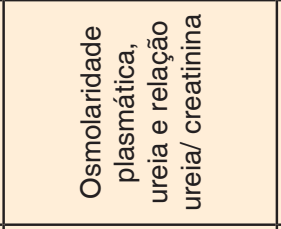 & 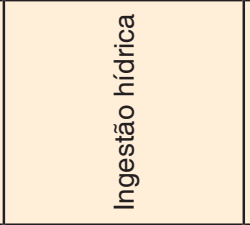 & 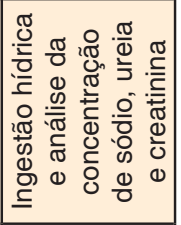 \\
\hline $\begin{array}{l}0 \\
\stackrel{2}{a} \\
\stackrel{\circ}{E}\end{array}$ & 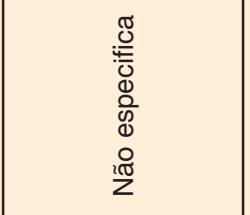 & 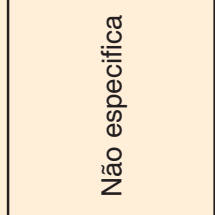 & 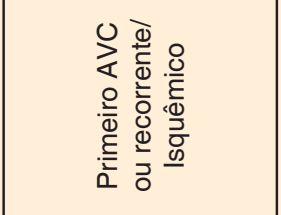 & 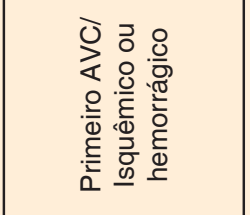 & 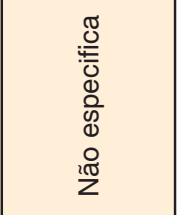 \\
\hline 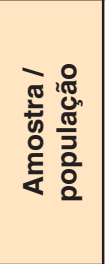 & 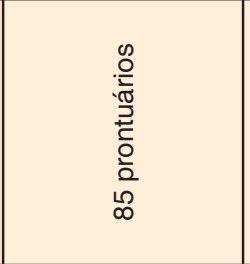 & 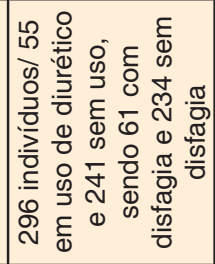 & 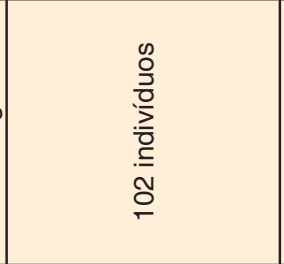 & 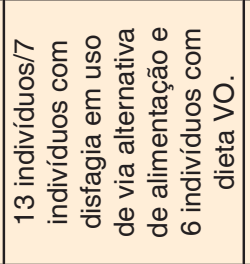 & 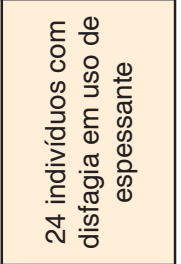 \\
\hline 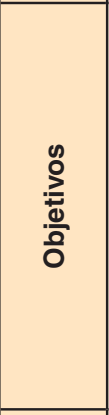 & 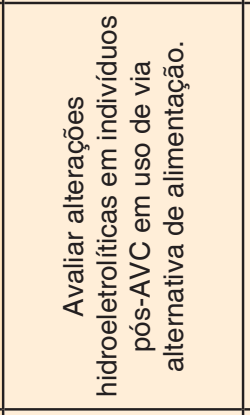 & 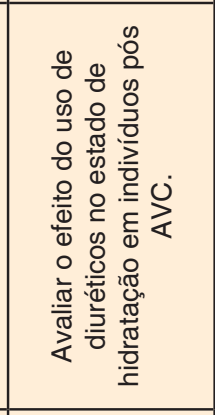 & 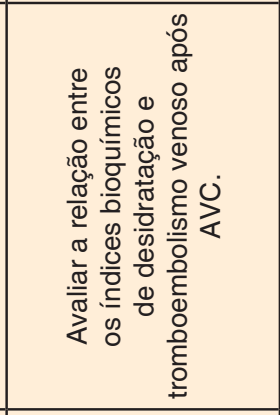 & 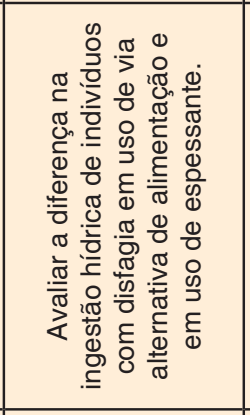 & 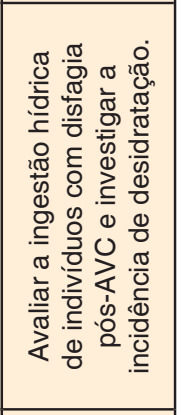 \\
\hline 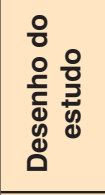 & 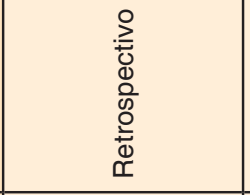 & 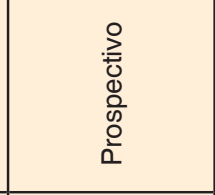 & 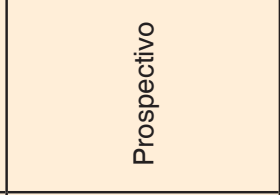 & 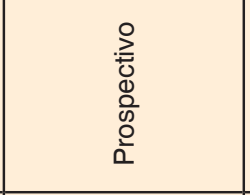 & 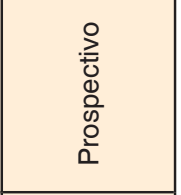 \\
\hline 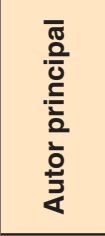 & 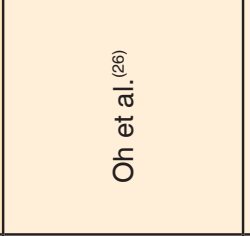 & 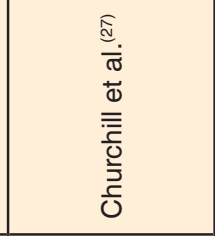 & 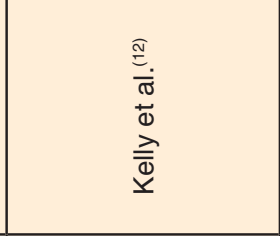 & 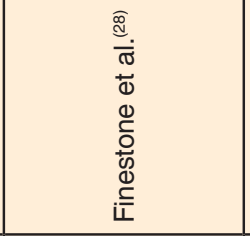 & 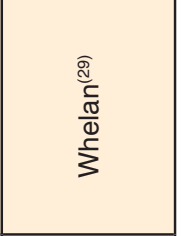 \\
\hline$\stackrel{\circ}{\frac{c}{4}}$ & \&্ণ & ষ্ণ & ষ্ণ & $\overline{\grave{Q}}$ & $\overline{\stackrel{\sim}{~}}$ \\
\hline
\end{tabular}


gravidade específica da urina $(n=2)^{(24,25)}$, coloração da urina $(n=1)^{(24)}$, ingestão hídrica $(n=3)^{(22,28,29)}$, balanço hídrico $(n=1)^{(26)}$, bioimpedância elétrica $(n=1)^{(21)}$, avaliação clínica $(n=1)^{(24)}$ e análise de eletrólitos isolados $(n=4)^{(12,26,27,29)}$. Em 44\% dos estudos selecionados, verificou-se associação de 2 ou mais métodos de avaliação do estado de hidratação ${ }^{(12,17,21,24-27,29)}$. Dentre os estudos que utilizaram apenas um método de avaliação, a relação BUN/creatinina apresentou maior prevalência ${ }^{(6-8,17-20)}$.

Em relação à frequência de avaliações do estado da hidratação, verificou-se que a maioria dos estudos $(n=13)^{(8,12,17-20,22-28)}$ realizou mais de uma avaliação ao longo da internação hospitalar, com média de 5 avaliações, sendo a primeira realizada sempre no momento da admissão hospitalar e as demais, durante a internação ou no momento da alta hospitalar.

$\mathrm{O}$ aumento dos valores da relação BUN/creatinina foi relacionado às alterações no estado de hidratação, quando superior a $15: 1(n=3)^{(7,8,25)}, 20: 1 \quad(n=2)^{(19,20)}$ e $25: 1 \quad(n=2)^{(6,27)}$. Já a relação ureia/creatinina foi considerada indicativa de desidratação, quando superior a 60:1 $(n=1)^{(24)}$ ou 80:1 $(n=2)^{(12,23)}$.

Em pacientes com disfagia orofaríngea, em uso de espessante para a modificação da consistência alimentar, os resultados obtidos demonstraram correlação com a diminuição da ingestão hídrica durante o período de internação hospitalar ${ }^{(19,20,22,28,29)}$.

A utilização da bioimpedância elétrica e a análise da gravidade específica da urina associada à sua coloração não foram considerados métodos eficazes na predição da desidratação em pacientes após AVC, quando comparados com a osmolaridade plasmática e a relação ureia/creatinina respectivamente ${ }^{(21,24)}$. No entanto, um estudo verificou possível associação entre gravidade específica da urina superior a 1.010 com AVC em evolução ${ }^{(25)}$.

Em outro estudo, o uso de diuréticos por pacientes com disfagia orofaríngea foi observado em $20 \%$ da amostra (11/55) e foi considerado fator de risco para a desidratação, quando avaliada a relação BUN/creatinina ${ }^{(27)}$.

Os valores dos principais eletrólitos (sódio, potássio, cloreto) não apresentaram variações significativas em pacientes com disfagia orofaríngea, após o início do uso de via alternativa de alimentação. Porém, houve aumento na porcentagem de $93 \%$ para $97,4 \%$ dos indivíduos com alteração nos valores da glicose ${ }^{(26)}$.

Índices bioquímicos indicativos de alteração no estado de hidratação, identificados em pacientes na fase aguda após o AVC, durante o período de internação hospitalar, estão diretamente associados ao desenvolvimento de trombose venosa ${ }^{(12)}$.

Com relação ao momento da admissão hospitalar, verificou-se prevalência de desidratação em 11\%, 34\% e 55\% dos pacientes após AVC e, ao longo da internação hospitalar, os índices de desidratação aumentaram para $36 \%, 46 \%$ e $66 \%{ }^{(8,12,19)}$.

\section{DISCUSSÃO}

A desidratação é usualmente encontrada em pacientes após AVC e está associada com a piora na evolução clínica, incluindo aumento da morbidade e mortalidade. Na fase inicial do AVC, a alteração no estado de hidratação pode ser consequência da diminuição da ingestão hídrica consciente, ou devido à presença de disfagia orofaríngea ${ }^{(24,28,29)}$.

No decorrer da internação hospitalar, é comum verificar a diminuição dos índices BUN/creatinina em pacientes após $\mathrm{AVC}$, porém, estudos demonstraram que aqueles com disfagia orofaríngea apresentam aumento dos índices e diminuição da ingestão hídrica nesse período, indicando piora do estado de hidratação ${ }^{(8,12,23,24)}$

Apesar de terem sido realizadas buscas nas principais bases de pesquisa científica relacionadas ao tema, foi encontrado um número reduzido de publicações que abordassem, especificamente, o diagnóstico/avaliação da desidratação em pacientes após AVC. Observou-se que a relação entre AVC, disfagia e desidratação ainda é pouco investigada, quando comparada, por exemplo, com a associação entre AVC, disfagia e desnutrição. Provavelmente, isto se deve à ausência de definição de um método padrão ouro de diagnóstico da desidratação e a grande heterogeneidade de métodos e valores de referência citados na literatura ${ }^{(6-8,13,14)}$.

Com relação aos critérios de inclusão e exclusão definidos, verificou-se que vários estudos não consideraram relevante o histórico de AVC prévio ${ }^{(6,7,12,14,17-20,22,25,26,28,30)}$.

Dentre as diversas técnicas de avaliação do estado de hidratação utilizadas na rotina clínica dos serviços de urgência e emergência, os métodos de análise da relação BUN/creatinina e da osmolaridade plasmática têm sido adotados com relativa frequência, em função da praticidade e rapidez ${ }^{(7)}$.

Em geral, a relação BUN/creatinina, a partir de 20:1, é considerada indicativa de alteração no estado de hidratação, porém, estudos recentes utilizaram índices de 15:1, uma vez que observaram significância estatística deste valor com AVC em evolução e piora nas condições clínicas ${ }^{(7,8,25)}$. Entretanto, graus variados de aumento da relação BUN/creatinina também foram observados em pacientes com hemorragia do trato gastrointestinal ou com catabolismo proteico excessivo, associado ao alto consumo de proteínas, queimaduras, febre e administração de corticoesteroides ${ }^{(6)}$.

Com relação ao momento da admissão hospitalar, verificou-se prevalência relativamente alta de desidratação em pacientes após AVC. Estudos correlacionaram os achados com aumento do risco de AVC cardioembólico ${ }^{(6)}$ e deterioração neurológica ${ }^{(6,25)}$. Ao longo da internação hospitalar, os índices de desidratação aumentaram, indicando piora na evolução clínica $^{(8,12,19,23,24)}$.

O uso de diuréticos por pacientes com disfagia orofaríngea grave que utilizam espessante foi considerado fator de risco para a desidratação, demonstrando que as alterações no padrão de deglutição podem comprometer o aumento da ingestão hídrica, geralmente necessária para compensar os efeitos do uso do diurético ${ }^{(27)}$.

Estudo recomendou ingestão hídrica diária mínima de $1500 \mathrm{ml}$ para compensar todas as perdas hídricas provenientes da urina, fezes e suor, em pacientes com peso entre $50 \mathrm{Kg}$ e $80 \mathrm{Kg}^{(19)}$. Entretanto, pacientes com disfagia orofaríngea, em uso de espessante, apresentaram ingestão hídrica em quantidade inferior ao recomendado (455 - $947 \mathrm{ml} /$ dia), sendo necessário, em alguns casos, o uso de complementação por via alternativa de alimentação ${ }^{(22,26,28)}$.

Observou-se alteração apenas nos níveis de glicose em pacientes com disfagia orofaríngea, após o uso de via alternativa de alimentação. Estudo anterior relatou que a hiperglicemia é uma complicação comum em pacientes em uso de via alternativa de alimentação ${ }^{(26)}$.

Índices bioquímicos indicativos de desidratação estão diretamente associados ao desenvolvimento de trombose venosa em indivíduos após AVC. Pesquisadores consideraram a possibilidade da presença de trombose venosa prévia à internação, porém, por meio da análise multivariada verificou-se tendência não significativa para a associação, ao segundo dia de internação hospitalar, e aumento dos índices no período entre o segundo 
e nono dia. Os resultados indicaram aumento da desidratação ao longo da internação e associação causal entre desidratação e trombose venosa ${ }^{(12)}$.

Foram incluídos neste estudo apenas dois artigos que utilizaram a análise da gravidade específica da urina, como método diagnóstico de desidratação em indivíduos após AVC ${ }^{(24,25)}$. Apesar da similaridade quanto à metodologia nos estudos citados, um estudo considerou o método ineficaz, quando comparado com a análise da relação ureia/creatinina ${ }^{(24)}$.

Apesar de a bioimpedância elétrica ser considerada um método rápido e não invasivo para estimar os compartimentos corporais $^{(20)}$, alguns autores não recomendam a utilização do método na mensuração das variações agudas de fluido corporal em indivíduos após AVC, uma vez que a bioimpedância elétrica demonstrou baixa acurácia diagnóstica, ao ser comparada com a análise da osmolaridade plasmática ${ }^{(21)}$.

Devido às diferenças metodológicas dos estudos selecionados para análise final, não foi possível realizar comparações, por meio de metanálise, entre os resultados encontrados. Além disso, o momento da avaliação da hidratação também foi diferente entre os estudos incluídos, pois alguns mencionaram os dados apenas em um momento, na admissão ou ao longo da internação.

Por meio desta revisão sistemática foi possível compreender a complexidade do processo de mensuração do estado de hidratação em pacientes após AVC e a associação com a disfagia orofaríngea. Estudos enfocando a associação entre AVC, desidratação e disfagia são de extrema relevância, visto a sua influência sobre a taxa de mortalidade e morbidade nesta população.

\section{CONCLUSÃO}

A desidratação durante o período de internação hospitalar está associada à gravidade do acidente vascular cerebral e à piora na evolução clínica. Apesar da impossibilidade de generalização dos resultados, devido à heterogeneidade dos estudos, observou-se a importância de estratégias de monitoramento do estado de hidratação, para prevenção de agravos à saúde durante o período de internação hospitalar, com consequente diminuição do tempo de permanência e do risco de reinternação. Porém, há necessidade de novos estudos que avaliem a relação da desidratação e do AVC com a disfagia orofaríngea.

\section{REFERÊNCIAS}

1. Jéquier E, Constant F. Water as an essencial nutrient: the physiological basis of hydration. Eur J Clin Nutr. 2010;64(2):115-23. PMid:19724292.

2. Ritz P, Berrut G. The importance of good hydration for day-today health. Nutr Rev. 2005;63(6):6-13. http://dx.doi.org/10.1301/nr.2005. jun.S6-S13. PMid:16028567.

3. Ganio MS, Armstrong LE, Casa DJ, McDermott BP, Lee EC, Yamamoto LM, et al. Mild dehydration impairs cognitive performance and mood of men. Br J Nutr. 2011;106(10):1535-43. http://dx.doi.org/10.1017/ S0007114511002005. PMid:21736786.

4. Armstrong LE, Ganio MS, Casa DJ, Lee EC, McDermott BP, Klau $\mathrm{JF}$, et al. Mild dehydration affects mood in healthy young women. J Nutr. 2012;142(2):382-8. http://dx.doi.org/10.3945/jn.111.142000. PMid:22190027.
5. Lieberman HR. Hydration and cognition: a critical review and recommendations for future research. J Am Coll Nutr. 2007;26(5, Suppl):555-61. http://dx.doi.org/10.1080/07315724.2007.10719658. PMid:17921465.

6. Akimoto T, Ito C, Kato M, Ogura M, Muto S, Kusano E. Reduced hydration status characterized by disproportionate elevation of blood urea nitrogen to serum creatinine among the patients with cerebral infarction. Med Hypotheses. 2011;77(4):601-4. http://dx.doi. org/10.1016/j.mehy.2011.06.044. PMid:21778021.

7. Schrock JW, Glasenapp M, Drogell K. Elevated blood urea nitrogen/ creatinine ratio is associated with poor outcome in patients with ischemic stroke. Clin Neurol Neurosurg. 2012;114(7):881-4. http:// dx.doi.org/10.1016/j.clineuro.2012.01.031. PMid:22333035.

8. Crary MA, Humphrey JL, Carnaby-Mann G, Sambandam R, Miller L, Silliman S. Dysphagia, nutrition, and hydration in ischemic stroke patients at admission and discharge from acute care. Dysphagia. 2013;28(1):69-76. http://dx.doi.org/10.1007/s00455-012-9414-0. PMid:22684924.

9. Mourão AM, Lemos SMA, Almeida EO, Vicente LCC, Teixeira AL. Frequência e fatores associados à disfagia após acidente vascular cerebral. CoDAS. 2016;28(1):66-70. http://dx.doi.org/10.1590/23171782/20162015072. PMid:27074192.

10. Logemann JA. Oropharyngeal dysphagia and nutritional management. Curr Opin Clin Nutr Metab Care. 2007;10(5):611-4. PMid:17693745.

11. Armstrong LE. Hydration assessment techniques. Nutr Rev. 2005;63(6):4054. http://dx.doi.org/10.1301/nr.2005.jun.S40-S54. PMid:16028571.

12. Kelly J, Hunt BJ, Lewis RR, Swaminathan R, Moody A, Seed PT, et al. Dehydration and venous thromboembolism after acute stroke. QJM. 2004;97(5):293-6. http://dx.doi.org/10.1093/qjmed/ hch050. PMid: 15100423.

13. Thomas DR, Cote TR, Lawhorne L, Levenson SA, Rubenstein LZ, Smith DA, et al. Understanding clinical dehydration and its treatment. J Am Med Dir Assoc. 2008;9(5):292-301. http://dx.doi.org/10.1016/j. jamda.2008.03.006. PMid:18519109.

14. Rodriguez GJ, Cordina SM, Vazquez G, Suri MF, Kirmani JF, Ezzeddine MA, et al. The hydration influence on the risk of stroke (THIRST) study. Neurocrit Care. 2009;10(2):187-94. http://dx.doi.org/10.1007/ s12028-008-9169-5. PMid:19051062.

15. Côrtes MG, Meireles AL, Friche AAL, Caiaffa WT, Xavier CC. O uso de escalas de silhuetas na avaliação da satisfação corporal de adolescentes: revisão sistemática da literatura. Cad Saude Publica. 2013;29(3):427-44. http://dx.doi.org/10.1590/S0102-311X2013000300003. PMid:23532280.

16. Littell JH, Corcoran J, Pillai VK. Systematic reviews and meta-analysis. Oxford: Oxford University Press; 2008. http://dx.doi.org/10.1093/ acprof:oso/9780195326543.001.0001.

17. Goroff H, Herzog L, Cardi R, Reding M. Use of oral hydration protocols for dysphagic patients following stroke. Rehabil Nurs. 2018;43(5):290-6. PMid:30168811.

18. Murray J, Scholten I, Doeltgen S. Factor contributing to hydration, fluid intake and health status of inpatients with and without dysphagia post stroke. Dysphagia. 2018;33(5):670-83. http://dx.doi.org/10.1007/ s00455-018-9886-7. PMid:29497831.

19. Crary MA, Carnaby GD, Shabbir Y, Miller L, Silliman S. Clinical variables associated with hydration status in acute ischemic stroke patients with dysphagia. Dysphagia. 2016;31(1):60-5. http://dx.doi. org/10.1007/s00455-015-9658-6. PMid:26497649.

20. Murray J, Doeltgen S, Miller M, Scholten I. Does a water protocol improve the hydration and health status of individuals with thin liquid 
aspiration following stroke? A randomized controlled trial. Dysphagia. 2016;31(3):424-33. http://dx.doi.org/10.1007/s00455-016-9694-x. PMid:26886370

21. Kafri MW, Myint PK, Doherty D, Wilson AH, Potter JF, Hooper L. The diagnostic accuracy of multi-frequency bioelectrical impedance analysis in diagnosing dehydration after stroke. Med Sci Monit. 2013;19:54870. http://dx.doi.org/10.12659/MSM.883972. PMid:23839255.

22. McGrail A, Kelchner LN. Adequate oral fluid intake in hospitalized stroke patients: does viscosity matter? Rehabil Nurs. 2012;37(5):252-7. http://dx.doi.org/10.1002/rnj.23. PMid:22949278.

23. Rowat A, Graham C, Dennis M. Dehydration in hospital-admitted stroke patients: detection, frequency, and association. Stroke. 2012;43(3):857-9. http://dx.doi.org/10.1161/STROKEAHA.111.640821. PMid:22156691.

24. Rowat A, Smith L, Graham C, Lyle D, Horsburgh D, Dennis M. A pilot study to assess if urine specific gravity and urine colour charts are useful indicators of dehydration in acute stroke patients. J Adv Nurs. 2011;67(9):1976-83. http://dx.doi.org/10.1111/j.1365-2648.2011.05645.x. PMid:21507048.

25. Lin LC, Fann WC, Chou MH, Chen HW, Su YC, Chen JC. Urine specific gravity as a predictor of early neurological deterioration in acute ischemic stroke. Med Hypotheses. 2011;77(1):11-4. http://dx.doi. org/10.1016/j.mehy.2011.03.012. PMid:21444157.

26. Oh H, Seo W. Alterations in fluid, electrolytes and other serum chemistry values and their relations with enteral tube feeding in acute brain infarction patients. J Clin Nurs. 2007;16(2):298-307. http:// dx.doi.org/10.1111/j.1365-2702.2005.01424.x. PMid:17239065.

27. Churchill M, Grimm S, Reding M. Risks of diuretic usage following stroke. Neurorehabil Neural Repair. 2004;18(3):161-5. http://dx.doi. org/10.1177/0888439004268163. PMid:15375276.

28. Finestone HM, Foley NC, Woodbury MG, Greene-Finestone L. Quantifying fluid intake in dysphagic stroke patients: a preliminary comparison of oral and nonoral strategies. Arch Phys Med Rehabil. 2001;82(12):1744-6. http://dx.doi.org/10.1053/apmr.2001.27379. PMid:11733894.

29. Whelan K. Inadequate fluid intakes in dysphagic acute stroke. Clin Nutr. 2001;20(5):423-8. http://dx.doi.org/10.1054/clnu.2001.0467. PMid:11534937.

30. Fleiss JL, Levin B, Paik MC. Statistical methods for rates and proportions. New York:Wiley;1981. 